\title{
MP Systems Approaches to Biochemical Dynamics: Biological Rhythms and Oscillations
}

\author{
Vincenzo Manca \\ University of Verona \\ Department of Computer Science \\ Strada Le Grazie, 15 \\ 37134 Verona, Italy
}

\begin{abstract}
Metabolic P systems are a special class of $\mathrm{P}$ systems which seem to be adequate for expressing biological phenomena related to metabolism and signaling transduction in biological systems. We give the basic motivation for their introduction and some ideas about their applicability to some basic biological oscillators.
\end{abstract}

\section{Introduction}

P systems were introduced as a new computation model, inspired by biology [31, 33], where multisets and membranes are the two main ingredients. The theory of $\mathrm{P}$ systems has grown very fast by studying different kinds of evolution rules and strategies. Important mathematical results have been established on the computational power of different kinds of $\mathrm{P}$ systems and on their relationships with other computational models $[33,36]$. The state of a $\mathrm{P}$ systems is the multisets of objects present inside each membrane. The passage from a state to another is produced by the application of rules (a set of rules for each membrane) which act, independently in each membrane, and typically, are applied in a maximal parallel way. This means that a maximal set of rules which are applicable is chosen and applied in a parallel way.

The $\mathrm{P}$ system paradigm has also been used to mathematically model several biomolecular phenomena acting at the cellular level, such as trans-membrane transport and communication [29,30], consumption of energy [16,32] and even more specific biological processes $[15,4,2,9]$.

Early attempts of symbolic descriptions of metabolic processes were initiated by the author, approximately, ten years ago $[23,24]$. In these papers some primitive notions of membrane systems were considered, but the use of logical formulae driving metabolite concentrations made them too general for expressing biological situations in a significant way. The theory of $\mathrm{P}$ systems was crucial in two important steps toward a new symbolic model of a metabolic system. A first step was the dynamical perspective in the study of $\mathrm{P}$ systems, introduced in [3], where the dynamical patterns of $\mathrm{P}$ systems were the main focus of investigation. A second step was the introduction of a molar perspective, borrowed from chemistry, with an abstract notion of "reaction strength" as a parameter able to 
regulate the cooperation/competition among the rules of $\mathrm{P}$ systems [25]. In fact, in a very first approximation, a cell is a membrane system, and its functioning is determined by all the types of molecules inside it, by the amount of molecules of these types, and by the cell compartments where they are located [1]. Therefore, it is of great importance to define a method for computing the evolution of a $\mathrm{P}$ system that is directly meaningful with respect to biochemical reactions. In this perspective, a transformation $A A \rightarrow B C$ is better read in chemical terms, as something which expresses the following prescription: "two moles of $A$ produce one mole of $B$ and a mole of $C$ ". Here a mole is a conventional population unit like a battalion, a company, a brigade, which is not conceived in an absolute way, as it happens in the classical chemical setting ( 1 mole $\approx 6.02 \times 10^{23}$ molecules), but it is relative to the specific system. If we fix the number of objects of a mole, then the dimension of a multiset is expressed, in terms of moles, by a rational number.

When many reaction are working together, a competition among reactions needing the same kinds of reactants, is better expressed by a notion of reaction unit. For example, a rule such as $A A \rightarrow B C$ should reasonably say that $2 m$ objects of type $A$ have to be consumed by the rule, and $m$ objects $B$ plus $m$ objects $C$ have to be produced by it. The crucial point of this discussion is "how has the number $m$ to be calculated" in order to reproduce adequately a given biochemical process?" This problem becomes more difficult that it may appear at a first glance. Such a number depends on the relative strength of a rule with respect to the other rules which are competing with it for the same reactants. We call this strength reactivity, and in general, it depends on the current state of the system.

MP systems [27] formalize these intuitions by considering $\mathrm{P}$ systems with a particular deterministic procedure for computing their evolution. This procedure, called MP Algorithm, shortly MPA [28], aims at capturing the salient chemical mechanisms that are responsible of the dynamics of a wide class of biomolecular processes. We have shown that MP systems effectively model the dynamics of several biochemical processes: the Belousov-Zhabotinsky reaction (Brusselator) [6, 8], the Lotka-Volterra dynamics [25, 7,6, 8, 13], a Susceptibleinfected-recovered epidemic [6], the Leukocyte selective recruitment in the immune response [15,6], the Protein Kinase C Activation [8], Circadian rhythms [12], and Mitotic cycles [28]. Other phenomena under investigation concern Cdc25A degradation in tumor processes, an oscillatory circuit that includes Protein Kinases ERK2 and PK [22] and the intercellular communication which occurs in Dictyostelium discoideum. This organism is an amoeba very important in developmental biology, which may switch from unicellular to multicellular stages (isolated and collective phases) by means of a periodic chemical mechanism, similar to hormonal communications in higher organisms [18]. A project, of interest in the search for biological energy sources, intends to apply MP systems to the analysis of specific metabolisms occurring in microbial fuel cells. From a more theoretical point of view, interesting relationships were stated between MPA and ODE (ordinary differential equations) [14]. In the section 3, we show 
the relevance of these theoretical results in relation to an example of biological modeling [17].

In the analysis of MP systems and of their applications an important role is played by MP graphs [28], which we will briefly outline, and which yield an immediate formulation of the structural aspects of MP systems in a style similar to other graphical representations in signal transduction networks and metabolic pathways $[20,35]$.

\section{Metabolic P Systems}

MP systems are deterministic $\mathrm{P}$ systems where i) the state of the system, at each time instant, is given by the amount of matter that is assigned to any (chemical) substance present in the system, and ii) the transition to the next state (after some specified interval of time) is calculated according to some mass partition strategy, that is, the available matter of each substance is partitioned among all reactions which need to consume it. The policy of matter partition is regulated at each instant by some real values, say them reactivities, which represent the strength of any reaction.

The definition we give here of MP systems is similar, but different to those given in our preceding papers on this subject. In the present form it seems more appropriate to the further theoretical and experimental development of these systems, especially in the process of providing models from the data of biological observations.

A discrete multiset over an alphabet $T$ is a function from $T$ to the set $\mathbb{N}$ of natural numbers. A continuous multiset over an alphabet $T$ is a function from $T$ to the set $\mathbb{R}$ of real numbers. As it is customary in $\mathrm{P}$ systems, we will adopt the string notation for discrete multisets. Sometimes it is useful to use the symbol + for concatenation, in order to stress that in multisets concatenation is commutative, that is, when a string denotes a multiset, the order of its symbols is not relevant (see [33] for more details on $\mathrm{P}$ systems notations) and, for any string $\alpha$, we write $X \in \alpha$ for saying that $X$ is a symbol occurring in $\alpha$. The set $Q$ of states over an alphabet $T$ are the continuous multisets over $T$. The passage from discrete to continuous states is motivated by the use of moles for determining the mass associated to each symbol of $T$.

The notion of MP system we consider here should be better identified by that of zero level MP system, because only one membrane is considered.

Definition 1 (MP System). An MP system is a construct

$$
M=\left(T, R, F, \nu, \mu, \tau, q_{0}\right)
$$

in which

$-T$ is a finite set of symbols;

$-R$ is a finite set of rules, i.e., pairs of discrete multisets over $T$ (represented, as usual, in the arrow notation); 
- $F$ is the set of reaction maps, such that $F=\left\{f_{r} \mid r \in R\right\}$, where $f_{r}: Q \rightarrow$ $\mathbb{R}$. Very often the reactivity $f_{r}(q)$ in the state $q$ depends only on the mass associated to some of the symbols of $T$. For this reason, it is convenient to introduce a real variable $x=q(X)$ to any symbol $X \in T$. We write $f_{r}(x, y, \ldots)$ to explicitate the variables $x, y, \ldots$ which $f_{r}$ depend on, and in this case, $\gamma_{r}$ denotes the string $X Y \ldots$;

- $\nu$ is a natural number which specifies the value of a (conventional) mole of $M$;

- $\mu$ is a function which assigns to each $X \in T$, the mass $\mu(X)$ of a mole of $X$, with respect to some measure unit;

$-\tau$ is the temporal interval between two consecutive states;

- $q_{0}$, the initial state of $M$, an element of $Q$.

The temporal evolution of an MP system $M$ is calculated by means of a metabolic difference operator $\Delta_{q}$, which provides for any state $q \in Q$ a function

$$
\Delta_{q}: Q \rightarrow \mathbb{R}
$$

such that, for every $X \in T$, the state following $q$ in the temporal evolution of $M$ is given by $q(X)+\Delta_{q}(X)$.

Two assumptions are fundamental in the definition of reaction rules and reaction maps used by MPA, which directly relate to the perspective of mass partition strategy adopted for MP systems evolution.

Principle 1 (Transparency) In any MP system, for every $X \in T$, a rule $r_{X}$ is present, which is called transparent rule for the substance $X$, and such that $X \rightarrow X$. The reactivity of a transparent rule $r_{X}$, in a given state, indicates the inertia of substance $X$ to be transformed into other substances.

Principle 2 (Creativity) Any input rule $r$ of type $\lambda \rightarrow X$ is assumed to be, implicitly, transformed into a rule $\lambda_{r} \rightarrow \lambda_{r} X$ where $\lambda_{r}$ is a new symbol in $T$, say it the imput symbol of $r$. This means that a sort of input gate, as a container of a given capacity of $X$, is assumed to feed the system from the outside, at a rate depending on the reactivity of the input rule. This capacity determines the creativity of the rule $\lambda \rightarrow X$, as the maximum value of elements $X$ that can enter into the system at each evolution step.

The value of transparency of each element of $T$ (possibly extended with input symbols), and the value of the creativity of input rules are very important parameters for the evolution of MP system according to the strategy we are going to define.

In order to define our MP algorithm, which formalizes the intuition given at beginning of Section 2, we use the following notation from [28], that will be adopted in the rest of the paper and it will be always related to a metabolic system $M=\left(T, R, F, \nu, \mu, \tau, q_{0}\right)$. 


\section{Definition 2 (MP Notation).}

- Each $r \in R$ is denoted by $r: \alpha_{r} \rightarrow \beta_{r} ; \alpha_{r}$ identifies the multiset of the substrates of $r$, and $\beta_{r}$ identifies the multiset of the products of $r$;

$-h_{r}(X)$ is the number of occurrences of $X$ in $\alpha_{r}$;

- $g_{r}(X)$ is the number of occurrences of $X$ in $\beta_{r}$;

- $R_{\alpha}(X)=\left\{r \in R \mid X \in \alpha_{r}\right\}$;

$-R_{\beta}(X)=\left\{r \in R \mid X \in \beta_{r}\right\}$;

$-R(X)=R_{\alpha}(X) \cup R_{\beta}(X)$;

- $\Pi\left(\alpha_{r}\right)=\prod_{X \in \alpha_{r}} q(X)^{h_{r}(X)}\left(\Pi\left(\alpha_{r}\right)=1\right.$ if $\left.\alpha_{r}=\lambda\right)$.

We assume that if $\alpha_{r}=\lambda$, then $\beta_{r} \in T$, and if $\beta_{r}=\lambda$ then $\alpha_{r} \in T$.

Definition 3 (MPA). The value of the metabolic difference operator $\Delta_{q}$ of an $M P$ system, in a state $q \in Q$ and on a symbol $X \in T$, is given by:

$$
\Delta_{q}(X)=\sum_{r \in R(X)}\left(g_{r}(X)-h_{r}(X)\right) \cdot u_{r}(q)
$$

where

$$
u_{r}(q)=\min \left\{w_{Y, q}(r) \frac{q(Y)}{h_{r}(Y)} \mid Y \in \alpha_{r}\right\}
$$

and, for every $Y \in T$

$$
K_{Y, q}=\sum_{r \in R_{\alpha}(Y)} f_{r}(q) \quad \text { and } \quad w_{Y, q}(r)=\frac{f_{r}(q)}{K_{Y, q}}
$$

where it is assumed $K_{Y, q} \neq 0$.

\section{MP Graphs and Biological Examples}

The $\mathrm{P}$ metabolic algorithm was proven adequate in many case of biological modeling we listed at end of section 1. Examples of biological models, formalized in terms of PM systems, will be collected in [5].

In an MP system two parts are clearly distinguishable: the signature and the quantities. The first part indicates the kinds of objects, the reaction and their regulation structure. The second part specifies the quantitative aspect which give meaning to the numbers which describe the evolution of systems. We represent the signature of metabolic $\mathrm{P}$ systems, in a way directly readable in terms of PM algorithm, by means of graphs. Similar graphical formalisms were developed in the context of complex reaction networks (SNA, Stoichiometric Network Analysis, and MCA, Metabolic Control Analysis [10,11,34]). Formally, a MP graph is a structure $G=(T, R, F, E, C)$ where:

- $T$ is the set of nodes representing types (we can think of each $t \in T$ as a container holding a certain amount of a peculiar kind of substance). Usually, we represent such kind of nodes as big circles labelled with the type of objects contained in it. 
$-R$ is the set of nodes representing biochemical reactions between types. We represent each of the nodes in $R$ as a full bullet and we label it with the name of the reaction represented by that node.

$-F$ is the set of nodes labelled by reaction maps. We represent such kind of nodes with full rectangles. These nodes are connected with a, possibly empty, set of circles (types) but they are also connected with exactly one bullet node.

- $E$ is a set of nodes presenting input or output gates. It contains two different kind of nodes: input gates and output gates. Both of them have the triangular shape, where input gates have an arc exiting from a triangle vertex, and output gates have an arc entering in a triangle edge.

$-C$ is a set of arcs between nodes. Edges are of two different kinds: plain edges or dashed edges.

i) Plain edges connect types to biochemical reactions, in particular they specify reactants and products of the reaction. Arcs connecting reactants to reactions are depicted as lines while arcs connecting reactions to products appear as arrows (oriented arcs).

ii) Edges which connect types with square nodes (reactivity nodes) are depicted as dashed lines, while edges which connect square nodes with bullets are depicted as dashed arrows (see Figure 2).

The components $E, C$ of an MP graph can be deduced by $R$ and $F$, therefore we can omit them when we specify a graph.

Figure 2 shows an MP graph related to the mitotic oscillator in amphibian embryos, which is an important case study reported in [17]. Mitotic oscillations are a mechanism exploited by nature to regulate the onset of mitosis, that is the process of cell division aimed at producing two identical daughter cells from a single parent cell. More precisely, mitotic oscillations concern the fluctuation in the activation state of a protein produced by $c d c 2$ gene in fission yeasts or by homologs genes in other eukaryotes. The model here considered focuses on the simplest form of this mechanism, as it is found in early amphibian embryos. Here (see Figure 1) Cyclin is synthesized at a constant rate and triggers the transformation of inactive $\left(M^{+}\right)$into active $(M)$ cdc2 Kinase, by enhancing the rate of a phoshatase $E_{1}$. Another Kinase reverts this modification. On the other hand a kinase $E_{3}$ elicits the transformation from the inactive $\left(X^{+}\right)$to the active $(X)$ form of a protease that degrades cyclin, and this activation is reverted by a phoshatase $E_{4}\left(E_{1}, E_{2}, E_{3}, E_{4}\right.$ are not indicated in the figure, $v_{i}, v_{d}, V_{1}, V_{2}, V_{3}, V_{4}$ denote rates of the processes). The activation of cdc2 kinase provides the formation of a complex known as M-phase promoting factor (or $M P F)$. The complex triggers mitosis and the degradation of cyclin leads to the inactivation of the cdc2 kinase that brings the cell back to the initial conditions in which a new division cycle can take place. In yeasts and in somatic cells the cell cycle is subject to the control of many checkpoints, but the mechanism based on the activation-inactivation of cdc2 kinase remains the same [1]. The following equations are the differential model of its dynamics, where $c, m, x$ are 
the percentages of $C, M, X$ respectively $(1-m, 1-x$ are the percentages of $M^{+}, X^{+}$respectively):

$$
\begin{aligned}
\frac{d c}{d t} & =v_{i}-v_{d} x \frac{c}{K_{d}+c}-K_{d} c \\
\frac{d m}{d t} & =V_{1} \frac{(1-m)}{K_{1}+(1-m)}-V_{2} \frac{m}{K_{2}+m} \\
\frac{d x}{d t} & =V_{3} \frac{(1-x)}{K_{3}+(1-x)}-V_{4} \frac{x}{K_{4}+x}
\end{aligned}
$$

Figure 3 is a solutions of these equations obtained by numerical integration for some value of parameters given in [17]. the MP graph of Figure 2 was deduced by Goldbeter's model by means of a procedure given in [14].

Circadian rhythms are biochemical cycles evoked by variations in the expression level of genes. Such variations give rise to a surprisingly robust biological clock, synchronized with daylight and performing a complete cycle about every 24 hours. In the model of Drosophila melanogaster, circadian rhythms involve the oscillation of the Period (PER) and Timeless (TIM) proteins. According to this model the genes coding for PER and TIM proteins are inhibited by the presence of a PER- TIM protein complex. This complex is constituted by the two proteins, in the cytosol, under certain conditions, then it migrates inside the nucleus where becomes a PER and TIM suppressor. Gene expression and suppression result in a negative feedback network of signal transduction that has been formalized by a non-trivial system of nonlinear differential equations, devised by J. Leloup and A. Goldbeter [21, 18]. A graphical scheme of the model is depicted in Figure 5 and a solution of differential model, for suitable values, is given in Figure 6. Also in this case we obtained similar solutions by using a suitable MP system deduced by the differential model of [18].

A general relationship between MP graphs and ODE holds. In fact, MP Graphs transform naturally into ODE systems according to the mass principle, on which differential models are based on. The amount of a products generated by a reaction is proportional to the product of quantities of substrates (considered with their multiplicity). This idea is formalized by the following definition where the MP notation 2 is assumed, $x$ is the real variable $q(X)$, and $x^{\prime}$ denotes the derivative of variable $x$ with respect to time.

Definition 4 (MP-ODE Transformation). Let $G=(T, R, F)$ be an $M P$ graph. For every $X \in T$, let $x$ be the real variable associated to $X$, then the following is the ODE-transformed of $G$ :

$$
x^{\prime}=\sum_{r \in R}\left\{g_{r}(X)-h_{r}(X)\right\} f_{r}(q) \Pi\left(\alpha_{r}\right)
$$

The following classes of MP systems play an important role in the relationship between ODE and MP systems.

Definition 5 (Non-cooperative MP System). A non-cooperative MP system is an MP system whose rules are non-cooperative, e.g., $\alpha_{r} \in T$ for every rule $r$ of the system. 
Definition 6 (Uniformly Transparent MP System). For some $\phi \in \mathbb{R}$, an $M P$ system is $\phi$-uniformly transparent if, the reaction map of any transparent rule of the system has the same constant value $\phi$ in any possible state.

The following results can be proved as generalizations of those proved in [14].

Proposition 1. Given an ODE, we can find (in many possible manners) an $M P$ graph having the given ODE as its ODE transform.

Theorem 3. The computation of a non-cooperative $\phi$-uniformly transparent $M P$ system converges, as $\phi \rightarrow \infty$, to the solution provided by the ODE system obtained by using MP-ODE transformation.

Theorem 4. For any MP system $M$, there exists a non-cooperative MP system $M^{\prime}$ having the same ODE transform of $M$.

Corollary 1. Approximate solutions of autonomous ODE which describe metabolic systems can be solved by computing the evolution of suitable MP systems.

Figure 4 shows an MP solution, obtained by using the a noncooperative system having as ODE-transform just the ODE 1. The similarity with Golbeter's solution 3 is really impressive and confirms the validity of the previous theorems, in a very significant biological model.

\section{Conclusions}

In many cases we were able to translate classical differential models into MP systems which provided similar results. Moreover, we showed that, under suitable hypotheses, this translation can be done in a systematic way [14], based on general relations on the two principles underlying these dynamical models: the differential ones assuming a time partition strategy and the MP ones assuming a mass partition strategy.

Evolutions of MP systems are discrete dynamics where important dynamical concept could be investigated in the specific perspective of biomolecular dynamics. In fact, the approach developed in [26] could suggest useful criteria in the classification of dynamical features of biological relevance.

MP systems have several computational advantages with respect to the differential models, but their most important aspect is their direct biological meaning and their structure where the reaction level and the regulation level are clearly interconnected but separated.

From the three principles underlying MPA and from the definition 3, it follows that the reactivities of rules in any state are univocally related to their reaction units and therefore to the variations of substances (from a state to the next one). It would be interesting to find procedures that, under suitable hypotheses, could be able to recover from the knowledge of such variations the reaction units and then the reactivities, and finally, from the reactivities in different states, the reaction maps of rules. 
The search for MP systems where these procedures can be defined, and computationally treated, is the main problem to solve for a systematic applications of MP systems to complex dynamics. Without this possibility the construction of models is a very difficult task, which can be developed only with specific strategies depending on the particular cases.

An important aspect for future developments of our approach is the possibility to build directly a model from the data coming from the observation of biological phenomena. If we show that this task can be done in a systematic and efficient way, then MP systems will give a real useful instrument in the modeling of biological systems. The future development of theory and applications of these systems will tell us whether, or to which extent, they will satisfy this expectation.

\section{References}

1. B. Alberts and M. Raff. Essential Cell Biology. An introduction to the molecular biology of the cell. Garland Science, New York, 1997.

2. F. Bernardini and M. Gheorghe. Cell communication in tissue P systems: universality results. Soft Computing, 9(9):640-649, 2005.

3. F. Bernardini and V. Manca. P systems with boundary rules. In Proc. 3rd Workshop on Membrane Computing, LNCS 2597, 107-118, 2002. Springer.

4. D. Besozzi and G. Ciobanu. A P system description of the sodium-potassium pump. In G. Mauri, G. Păun, M. J. Pérez-Jiménez, G. Rozenberg, and A. Salomaa, editors, Membrane Computing, 5th International Workshop, WMC 2004, LNCS 3365, 210-223. Springer, 2005.

5. L. Bianco. Membrane Models of Biological Systems, PhD Thesis, University of Verona, in preparation.

6. L. Bianco, F. Fontana, G. Franco, and V. Manca. P systems for biological dynamics, in [9], pages 81-126. 2006.

7. L. Bianco, F. Fontana, and V. Manca. Reaction-driven membrane systems. In L. Wang, K. Chen, and Y.-S. Ong, editors, Advances in Natural Computation, First International Conference, ICNC 2005, Changsha, China, August 27-29, 2005, Proceedings, Part II, LNCS 3611, 1155-1158. Springer, 2005.

8. L. Bianco, F. Fontana, and V. Manca. P systems with reaction maps. International Journal of Foundations of Computer Science, 17(1):27-48, 2006.

9. G. Ciobanu, G. Pău, and M. J. Pérez-Jiménez, editors. Applications of Membrane Computing. Springer, Berlin, Germany, 2006.

10. B. L. Clark. Stability of Complex Reaction Networks. Adv. Chem. Phys., 43, 1-216, 1983.

11. D. A. Fell. Metabolic control analysis: a survey of its theoretical and experimental development. Biochemistry J., 286:313-330, 1992.

12. F. Fontana, L. Bianco, and V. Manca. P systems and the modeling of biochemical oscillations. In R. Freund, G. Păun, G. Rozenberg, and A. Salomaa, editors, 6th Workshop on Membrane Computing (WMC6), LNCS 3850, 199-208. Springer, 2005.

13. F. Fontana and V. Manca. Predator-prey dynamics in P systems ruled by metabolic algorithm. Submitted. 
14. F. Fontana and V. Manca. Discrete Solutions of Differential Equations by Metabolic P Systems Submitted.

15. G. Franco and V. Manca. A membrane system for the leukocyte selective recruitment. In A. Alhazov, C. Martín-Vide, and G. Păun, editors, LNCS 2933, pages 180-189, Tarragona, Spain, July 2004.

16. R. Freund. Energy-controlled P systems. In G. Păun and C. Zandron, editors, Proc. Int. Workshop WMC-CdeA 2002, LNCS 2597, 247-260, Springer.

17. A Goldbeter. A Minimal Cascade Model for the Mitotic Oscillator Involving Cyclin and cdc2 Kinase. PNAS, 88(20):9107-9111, 1991.

18. A Goldbeter. Computational approaches to cellular rhythms. Nature, 420:238-245, 2002.

19. A. Goldbeter. Biochemical Oscillations and Cellular Rhythms. Cambridge University Press, New York, 2004.

20. H. Kitano. Computational systems biology. Nature, 420:206-210, November 2002.

21. J.C. Leloup and A. Goldbeter. A model for circadian rhythms in Drosophila incorporating the formation of a complex between the PER and TIM proteins. Journal of Biological Rhythms, 13:70-87, 1998.

22. M. Maeda, S. Lu, G. Shaulsky, Y. Miyazaki, H. Kuwayama, Y. Tanaka, A. Kuspa, W. Loomis, Periodic Signaling Controlled by an Oscillatory Circuit That Includes Protein Kinases ERK2 and PK. Science, 304, 875-304, 2004.

23. V. Manca. Rewriting and Metabolism: A logical Perspective. In G. Păun, editor, Computing with Bio-Molecules, Springer, 1998.

24. V. Manca and D. M. Martino. From String Rewriting to Logical Metabolic Systems. In G. Păun and A. Salomaa, editors, Grammatical Models of Multi-Agent Systems, Gordon and Breach Science Publishers, 1999.

25. V. Manca, L. Bianco, and F. Fontana. Evolutions and oscillations of P systems: Applications to biological phenomena. In G. Mauri, G. Păun, M. J. Pérez-Jiménez, G. Rozenberg, and A. Salomaa, editors, Membrane Computing, 5th International Workshop, WMC 2004, LNCS 3365, 63-84. Springer, 2005.

26. V. Manca, G. Franco, and G. Scollo. State Transition Dynamics: basic concepts and molecular computing perspectives. In M. Gheorghe, editor, Molecular Computational Models: Unconventional Approachers, Chapter 2., 32-55, Idea Group Inc. UK, 2005.

27. V. Manca. Topics and Problems in Metabolic P systems. In G. Păun and M. J. Pérez-Jiménez, editors, Proc. of the Fourth Brainstorming Week on Membrane Computing (BWMC4), Sevilla, Spain, Fenix Editora, 2006.

28. V. Manca, L. Bianco. Biological Networks in Metabolic P Systems. Submitted.

29. C. Martin-Vide, G. Păun, and G. Rozenberg. Membrane systems with carriers. Theoretical Computer Science, 270:779-796, 2002.

30. A. Păun and G. Păun. The power of communication: P systems with symport/antiport. New Generation Computing, 20(3):295-306, 2002.

31. G. Păun. Computing with membranes. J. Comput. System Sci., 61(1):108-143, 2000.

32. G. Păun, Y. Suzuki, and H. Tanaka. P systems with energy accounting. Int. J. Computer Math., 78(3):343-364, 2001.

33. Gh. Păun. Membrane Computing. An Introduction. Springer, Berlin, Germany, 2002 .

34. L. A. Segel and I. R. Cohen, editors. Design principles for the Immune System and Other Distributed Autonomous Systems. Oxford University Press, 2000.

35. E. O. Voit. Computational Analysis of Biochemical Systems. Cambridge University Press, 2000. 
36. The P Systems Web Page. http://psystems.disco.unimib.it

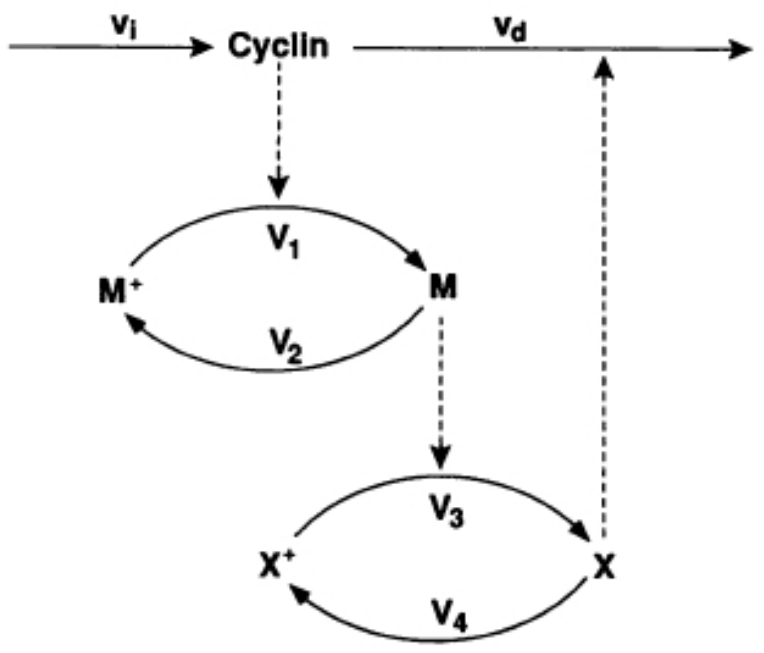

Fig. 1. The model provided by A. Goldbeter, from [17]. 


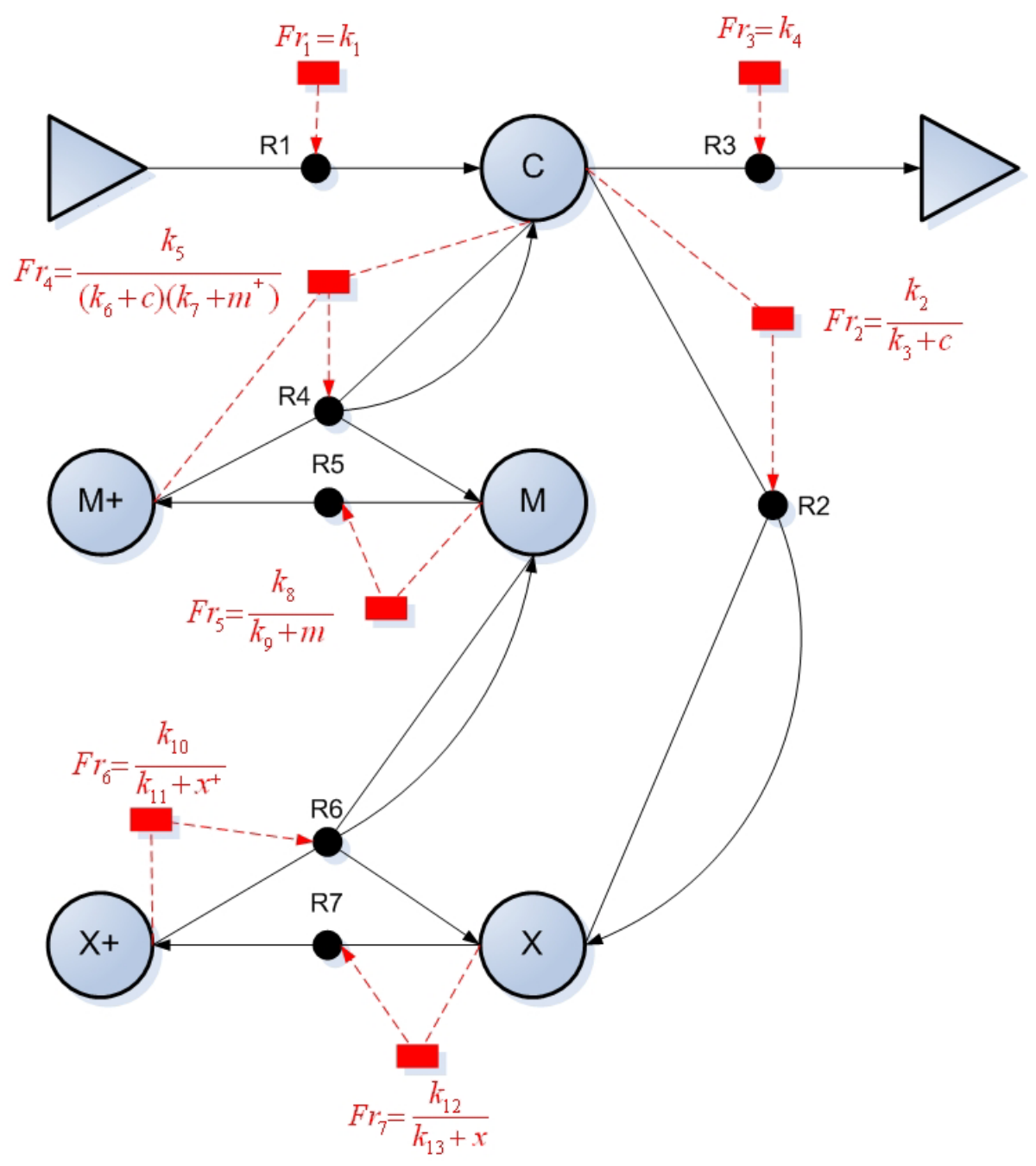

Fig. 2. A model of the mitotic oscillator of Figure 1 represented by a MP graph (from $[28])$ 


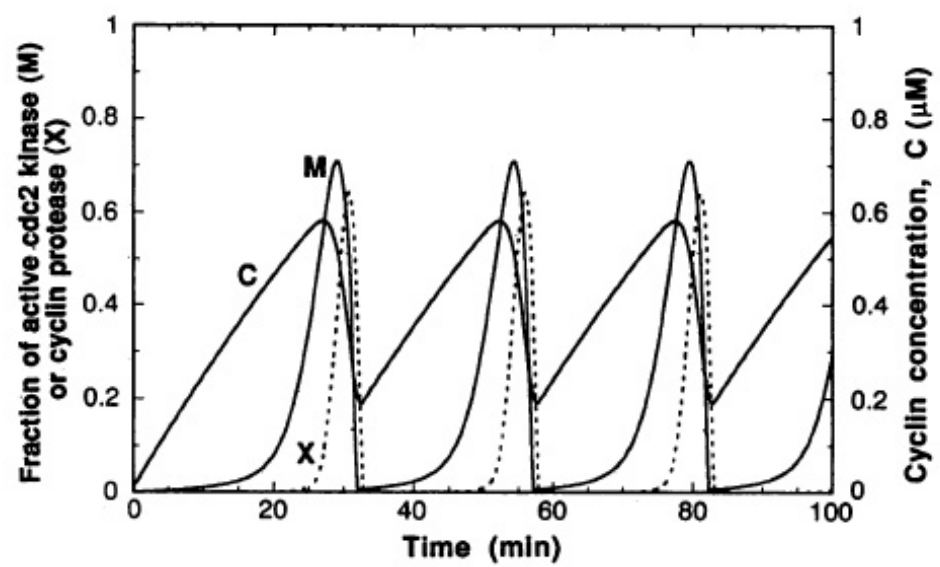

Fig. 3. A numerical solution of the set of differential equations (1) implementing the model provided by A. Goldbeter, from [17].

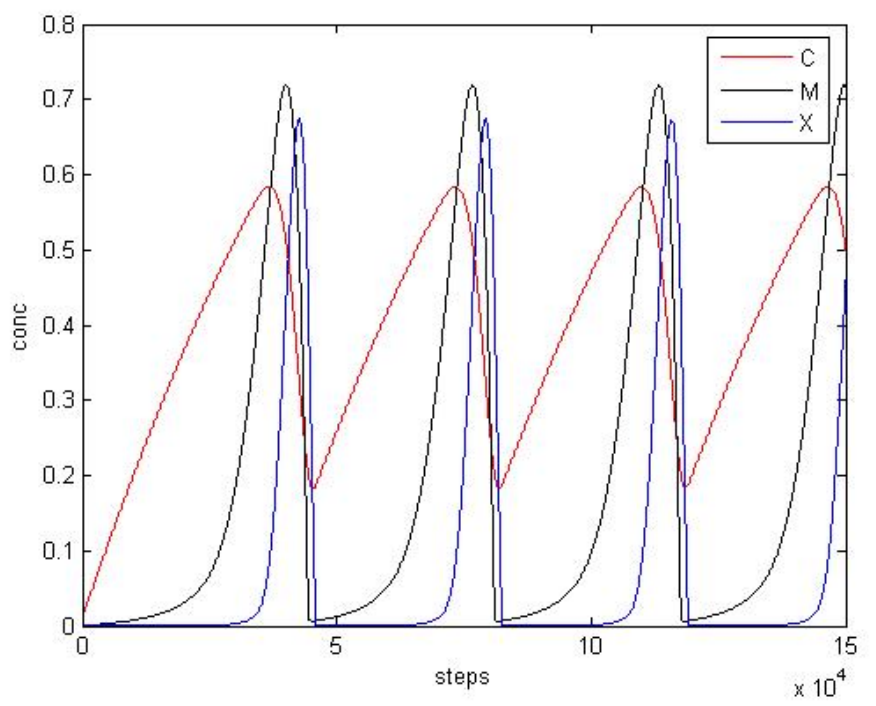

Fig. 4. The mitotic oscillator of Figure 1 computed means of an MP system evolution 


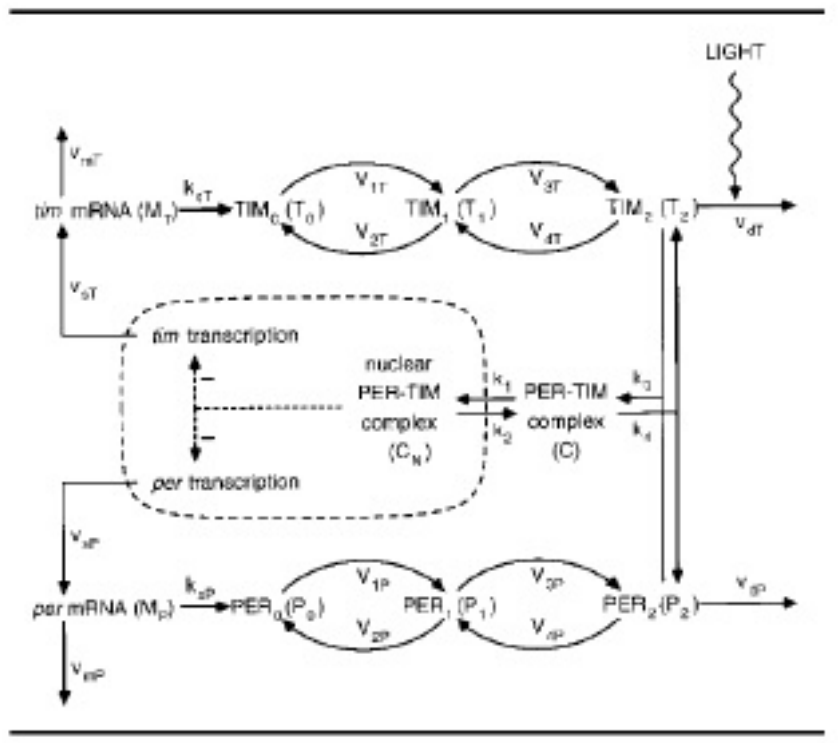

Fig. 5. Circadian rhythms in Drosophila, from [21].

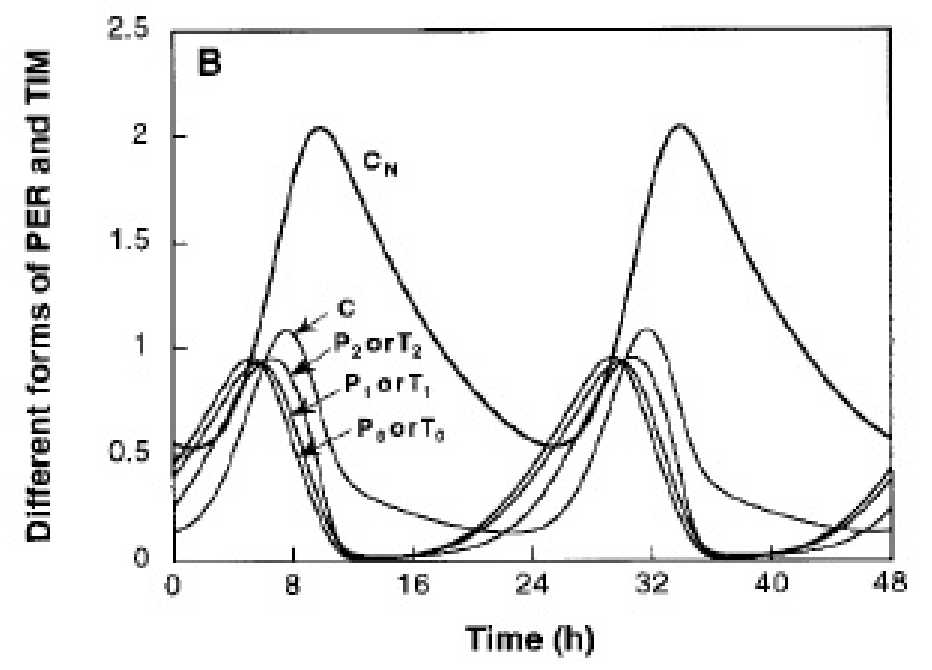

Fig. 6. Circadian rhythms in Drosophila: a numerical solution from [21]. 